\author{
Revista Minelor - Mining Revue \\ ISSN-L 1220-2053 / ISSN 2247-8590 \\ vol. 27, issue 4 / 2021, pp. 28-32
}

\title{
ANALYSIS OF A REPLACEABLE CUTTING TOOTH OF BUCKET CHAIN EXCAVATOR
}

\author{
Zoltán VIRÁG ${ }^{1 *}$, Sándor SZIRBIK ${ }^{2}$ \\ ${ }^{1}$ University of Miskolc, Institute of Mining and Geotechnical Engineering, Miskolc, Hungary, gtbvir@ uni-miskolc.hu \\ ${ }^{2}$ University of Miskolc, Institute of Applied Mechanics, Miskolc, Hungary, sandor.szirbik@uni-miskolc.hu
}

DOI: $10.2478 / \operatorname{minrv}-2021-0032$

Keywords: bucket chain excavator, cutting teeth, detachable joint, cutting load, FEA

Abstract: This paper briefly outlines the design of replaceable cutting teeth of bucket chain excavator, which are attached to a holder with a detachable joint. The description of the rock cutting process is very complex, so the investigation of the effect of lateral forces is complicated through cutting tests. We use accordingly numerical analysis to examine some segments of the cutting process. Our main objective is to present the finite element analysis of cutting teeth in which the linear increase of the lateral force is taken into consideration. The finite element analysis is a powerful technique, which is enabled to compute the stress and displacement distribution in cutting teeth. The simulation results have shown that the maximum stresses decrease if the lateral force increases. The geometry of the optimized cutting teeth will be safe under the given loading conditions.

\section{Introduction}

Bucket wheel and bucket chain excavators are used in open pit coal mining are subjected to excessive loads during cutting because of the interaction between the working face and the excavation components. High loads and unexpected shocks produce numerous damages to the excavator components leading to increased energy consumption and lower production rate. Radu et al. [1] performed a study of the forces acting on the shaft of the bucket wheel was conducted to reduce the mentioned issues and improve the quality of the excavation process.

An important and complex issue in mining is also rock cutting mechanization. The high cutting tooth wear and the high specific power demand are the long-standing problems of mining techniques. The new cutting tooth was designed to eliminate problems. Rock cutting tests were carried out, where winning experiments on the large sample from the mine were made using chisels modelling the in-plant winning chisels (cutting teeth), with cutting parameters close to reality [2].

To determine the geometric configuration of the cutting teeth and the cutting edges the knowledge of the mining technology is inevitable. The measurement data of cutting is collected and registered by a computer aided measurement system. On rock samples, we recorded 165 measurement cycles. In each measurement cycle, the following data and cutting characteristics were recorded: cutting direction, form, depth, average chip area, average cutting force $F_{V}$, average pressing force $F_{R}$ and average lateral force $F_{O}$. As a result of the measurements, a new cutting geometry was developed [2]. The experiments prove that it should pay attention to the wears and the geometry of cutting teeth because of the increasing of the cutting, pressing and lateral force. Considering the laboratory cutting test results the FEA was created for further investigations for replaceable cutting teeth of bucket chain excavators. The results lead to determine the most critical point of the cutting tool, which help us to select the proper high strength steel that tolerates the extreme loads.

* Corresponding author: Zoltán Virág, Assoc. Prof., University of Miskolc, Miskolc, Hungary, (H-3515 Miskolc-

Egyetemváros, gtbvir@uni-miskolc.hu) 


\section{Model geometry and loading}

To investigate the distribution of the main stresses in the cutting tooth, we set up a finite element model. Consider the solid model shown in Figure 1, consisting of a holder, apart from the cutting edge and an optimized cutting tooth. The cutting tooth is jointed into the holder as a removable piece. The tooth holder is a steel rectangular structural tube and of course, it is possible the remaining parts of the structure are made of different grades of steel.

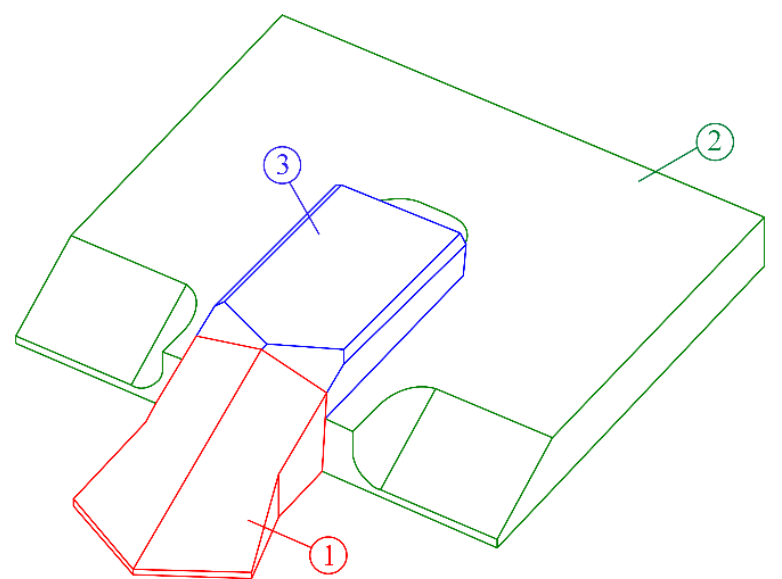

Fig. 1. Assembly of solid models (3 parts) 1 - cutting tooth, 2 - cutting edge, 3 - holder

The geometrical model of the tooth is illustrated in Figure 2. The material is assumed to be isotropic elastic: the material properties are Young's modulus of $\mathrm{E}=2.1 \times 10^{5} \mathrm{MPa}$ and Poisson's ratio of $v=0.3$. The finite element method, which is a very popular numerical technique, is used to numerically solve differential equations arising in engineering problems. The main concept of the technique is that the geometry of structure subdivides into non-overlapping small parts, so-called finite elements, which are implemented by the construction of a mesh. The conventional element types possess simple shaped geometry with well-defined stress displacement relationships. Thus, the sufficiently refined meshes need to ensure that the results from simulations are adequate. Accordingly, the cutting tooth is meshed into finite elements, which are 10-node tetrahedral elements.

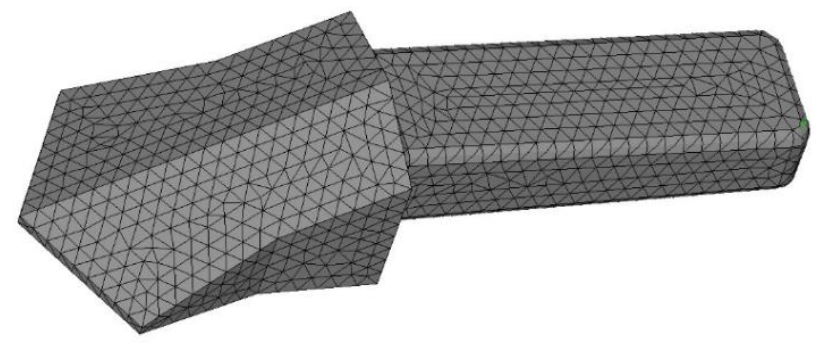

Fig. 2. FE model of cutting tooth

The aim of previous work [3] is to simulate the cutting load from real cutting conditions on the surface, which is painted with red colour in Figure 3. The resultant force from the pressure on the surface consists of three components: cutting force $F_{V}$, lateral force $F_{O}$ and a pressing force $F_{R}$.

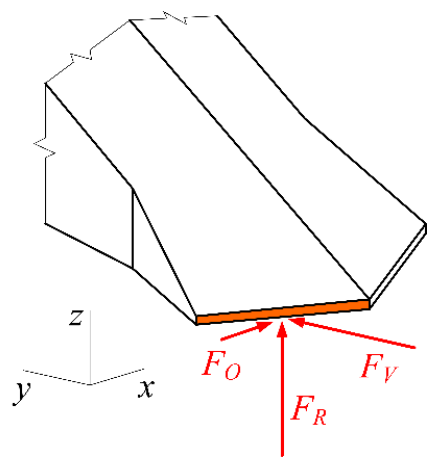

Fig.3. Cutting, lateral and pressing force 
The force components were determined by means of laboratory cutting experiments [2]. We assume that now the values of the resultant of cutting and lateral forces are $100 \mathrm{kN}$, and the pressing force is always 100 $\mathrm{kN}$. To investigate the rock cutting action for cutting forces in different directions, various direction angles are defined. The direction angle $\alpha$, which is measured in degrees positive clockwise between the direction of cutting force and the y-axis of the global coordinate system, specifies the magnitudes of lateral and cutting forces. Thus

$$
\begin{aligned}
& F_{R}=F=100 \mathrm{kN} \\
& F_{V}=F \cos \alpha \\
& F_{O}=F \sin \alpha
\end{aligned}
$$

are imposed on the appropriate surface as specified loads.

Because of the detachable contact joint, we should describe five contact pairs between the appropriate surfaces of the tooth and holder in the finite element analysis. The contact pair consists of the two contact surfaces. One of them in the pair is selected to be the contactor surface on the tooth and the other contact surface to be the target surface on the holder. For simplicity, the contact surfaces of the holder are regarded as stationary, rigid surfaces. It follows that these surfaces are used in our model to simplify the contact searching instead of modelling the holder as a solid structure. We assume that the coefficient of friction is equal to zero between the contact surfaces. Under these conditions, we should solve this contact problem by using the finite element method. Further details of contact problems are presented in [4], [5].

\section{Analysis results}

To preserve the integrity of the teeth, the maximum stresses should be kept under the proportional limit of the material. The finite element analysis helps accordingly to qualify the strength of the new cutting tooth. As appeared from the numerical simulation the maximum effective stress is about $900 \mathrm{MPa}$ and it occurs in front of the tooth (see Figure 4a-d). However, the stresses decrease quickly below $60 \mathrm{MPa}$ in the remaining part and so in the shank of the tooth.

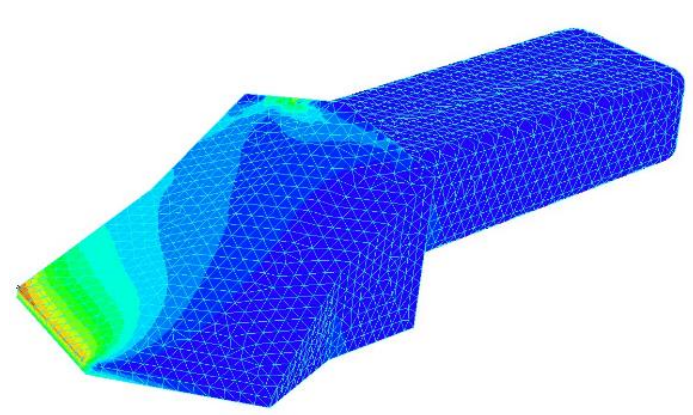

(a)

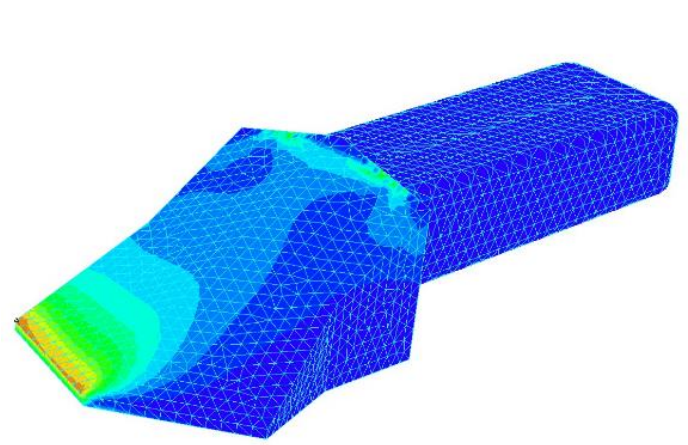

(c)
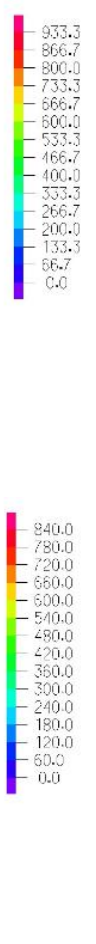

Fig. 4. Effective stress (von Mises) in the tooth:

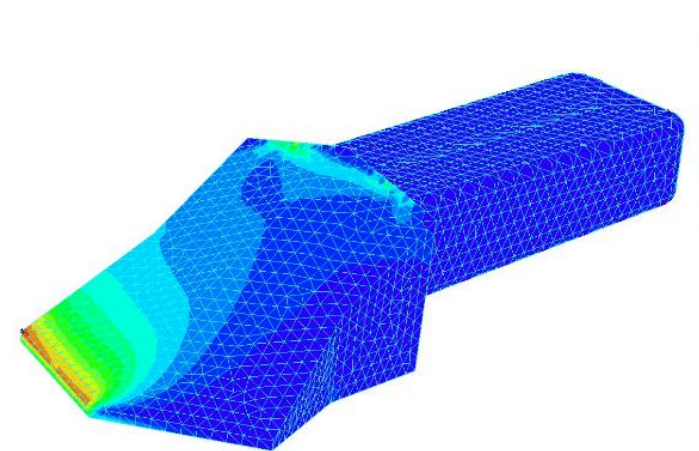

(b)

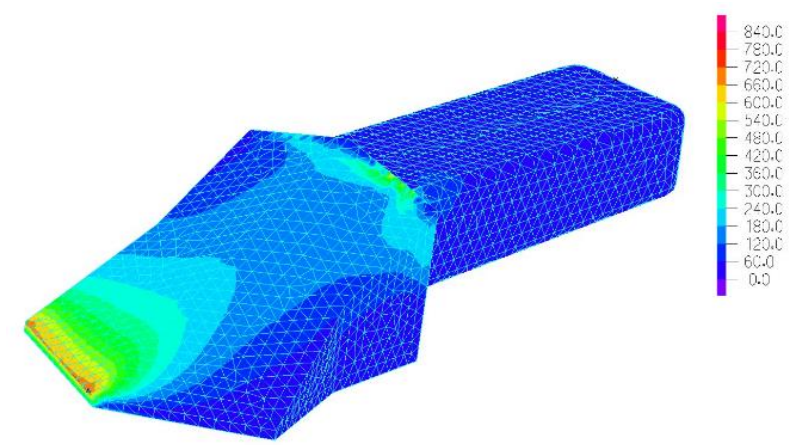

(d)

Figure 5a-d shows the magnitude of displacement, colour-coded at each point in the structure, to visualize the calculated deformation. The maximum displacement occurs also in front of the tooth, where the loading condition is imposed. The maximum value of deformation is $0.532 \mathrm{~mm}$ (see Figure 5d). In the remaining part, displacements decrease also quickly. 


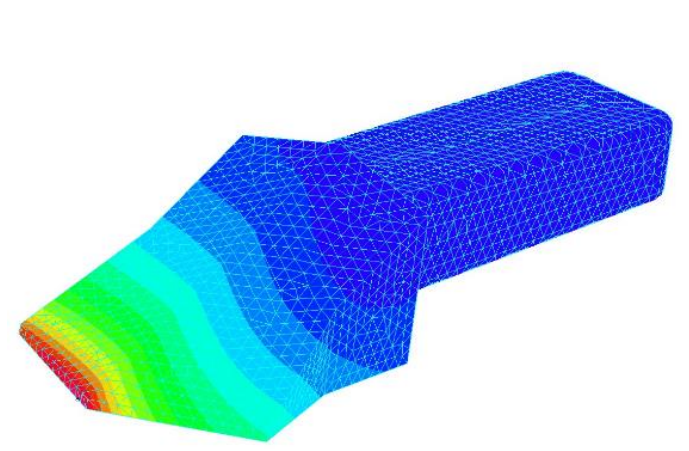

(a)

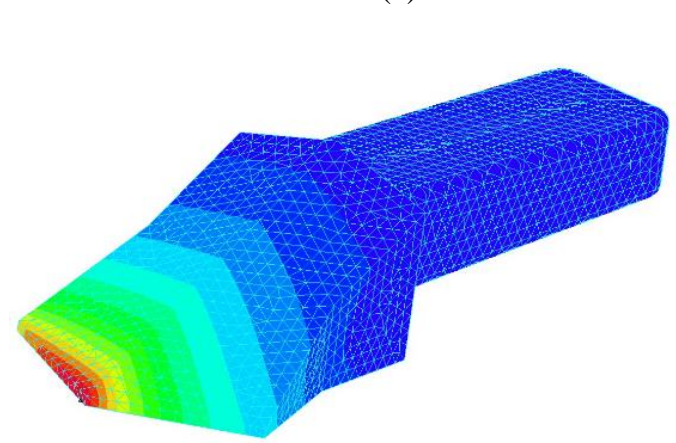

(c)
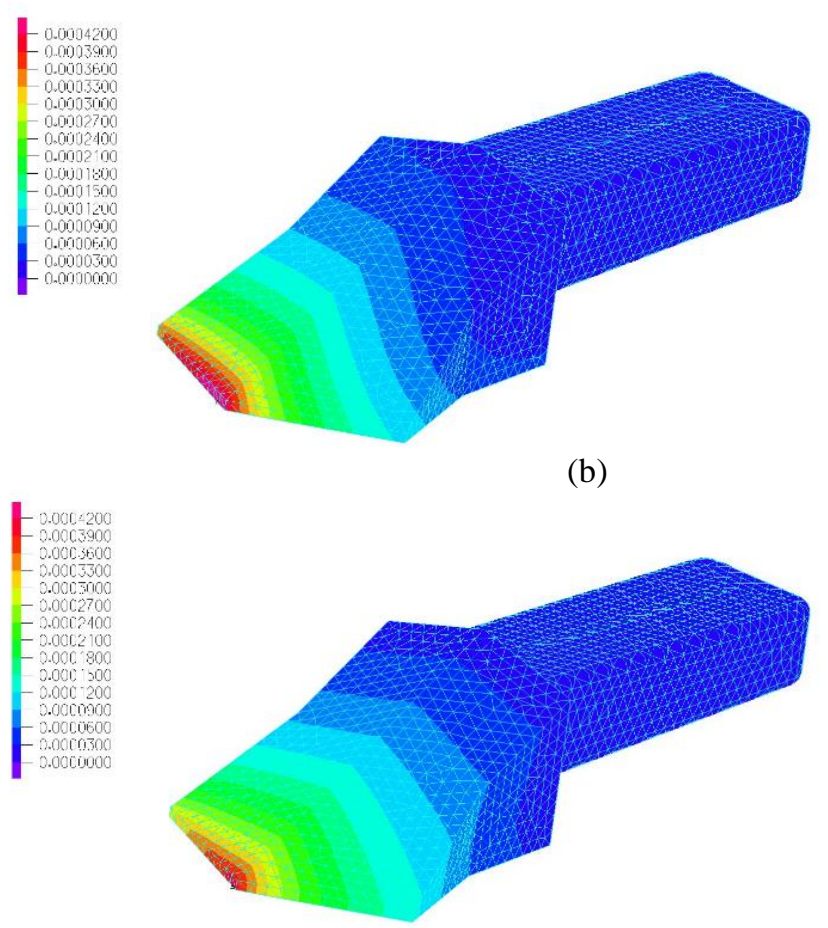

(d)

Fig. 5. Magnitude of displacement in the tooth: (a) $\alpha=0^{\circ}$; (b) $\alpha=10^{\circ}$; (c) $\alpha=20^{\circ}$; (d) $\alpha=30^{\circ}$

The corresponding results after changing direction angle are tabulated in Table 1, which contains the maximum effective stress $\sigma_{\max }$ and the maximum displacement $u_{\max }$. As mentioned previously, angle $\alpha$ is measured in degrees, and according to Table 1, four different values are employed, namely 0, 10, 20 and 30.

Table 1. Maximum values of numerical results

\begin{tabular}{|c|c|c|}
\hline $\boldsymbol{\alpha}$ & $\boldsymbol{\sigma}_{\max }[\mathbf{M P a}]$ & $\mathbf{u}_{\max }[\mathbf{m m}]$ \\
\hline $0^{\circ}$ & 933.3 & 0.426 \\
\hline $10^{\circ}$ & 884.9 & 0.389 \\
\hline $20^{\circ}$ & 850.3 & 0.422 \\
\hline $30^{\circ}$ & 829.3 & 0.532 \\
\hline
\end{tabular}

The simulation results show differences in the values of displacements. However, the stresses decrease if the angle increases. We remark that these results show good analogy with those obtained in the previous study [3], where the whole structure is modelled as a solid structure. Finally, the theoretical optimized cutting tooth for the bucket chain excavator is realized.

\section{Conclusions}

It is established that the development of cutting teeth improves by using finite element analysis that is applicable to compare easily different types of teeth. It leads to an assessment of the effect of the main parameters on the behavior of the geometric configuration of the cutting teeth. Consequently, the results of finite element analysis show that the head of the cutting tooth is the most critical point and so we can draw that high strength steel will be adequate because of the extreme loads. The FEA results can be useful in practice. Therefore, it is important to know that the cutting teeth are properly designed, made from proper materials, and constructed considering loading during their lifespan. 


\section{References}

[1] Radu, S. M., Popescu, F. D., Andras, A., Kertesz, I., Tomus O. B. 2018

Simulation and modelling of the forces acting on the rotor shaft of BWEs, in order to improve the quality of the cutting process, Annals of the University of Petrosani, Mechanical Engineering 20

[2] Ladanyi, G., Sumegi, I., Virag, Z. 2007

Laboratory rock cutting tests on rock samples from Visonta South Mine, Annals of the University of Petroşani, Mechanical Engineering, 9, pp. 209-218.

[3] Ladanyi, G., Virag, Z. 2016

Examining the bucket wheel excavator's bucket after renewal, Annals of the University of Petrosani: Mechanical Engineering 18, pp. 93-98.

[4] Bathe, K. J. 1996

Finite Element Procedures, Prentice-Hall, Inc., New Jersey.

[5] Wriggers, P. 2006

Computational Contact Mechanics, Spinger-Verlag Berlin Heidelberg.

This article is an open access article distributed under the Creative Commons BY SA 4.0 license. Authors retain all copyrights and agree to the terms of the above-mentioned CC BY SA 4.0 license. 